\title{
The Genetics Corner: The Positive Predictive Value of NIPT for 22q11 Deletion Syndrome Varies with the Indication
}

Nivedita Rajakumar, Subhadra Ramanathan, Robin D. Clark, MD

\section{Case Summaries}

\section{Patient 1}

A 31-year-old G4 P1 female was referred for prenatal genetic counseling at 28 w $3 \mathrm{~d}$ gestation for a fetal cardiac anomaly. A detailed anatomy scan at 20 weeks

gestation identified a tetralogy of Fallot with pulmonary atresia, confirmed by a fetal echocardiogram. The mother chose to have a cell-free fetal DNA (cff) non-invasive prenatal test (NIPT) that included screening for microdeletion syndromes. The NIPT (genome-wide counting method, QNATAL, Quest) reported:"high risk" for 22q microdeletion syndrome (fetal fraction 19.79\%; sensitivity $69-99 \%$ and PPV 75\%; Guy et al., 2019). The mother declined confirmatory diagnostic testing during the pregnancy. The baby boy was born at 38 weeks five days' gestation by planned induction of labor. Apgar scores were 8 and 8 .

Birth weight:

$3885 \mathrm{~g} \mathrm{(8} \mathrm{lb} 9 \mathrm{oz})$

Birth length:

$52 \mathrm{~cm}(20.47)$

Birth head circumference:

$33 \mathrm{~cm}(12.99 ")$

Postnatally, a chromosomal microarray confirmed a 2.5MB deletion at 22q11.21. Parental samples were normal, and the deletion was determined to be a de novo variant. He had cardiac surgery with unifocalization at nine months with plans for further cardiac surgery to repair pulmonary atresia. Other problems include bilateral hydroureteronephrosis, recurrent UTI, and an intradural arachnoid cyst of the spine. He is growing well with intact immune function, but global developmental delay affects his gross motor, fine motor, and speech.

\section{Patient 2}

A 12-month old female was referred to clinical genetics for confirmatory testing as cell-free fetal (cff) DNA screening testing during pregnancy was positive for 22q11.2 deletion syndrome. The pregnancy was detected at around 23 week's gestation, and NIPT was offered in place of routine maternal serum screening as the 25-year old mother was late to prenatal care. There were no fetal anomalies. NIPT (SNP-based method, Panorama, Natera) at 27 week 1 day's gestation reported a high risk for 22q11.2 deletion syndrome (fetal fraction $8.9 \%$, risk before test: $1 / 2000$, risk after test: $1 / 5$, sensitivity $90 \%(9 / 10)$, specificity $99.74 \%$ (389/390), false positive-rate $0.26 \%$; Ravi et al. 2018). Fetal ultrasound and echocardiogram exams were normal.

The baby was born at $39 w 6 d$ by NSVD.

Birth weight: $3885 \mathrm{~g}(8 \mathrm{lb} 9 \mathrm{oz})$

Birth length:

$54 \mathrm{~cm}(21.25 ")$
Birth head circumference: $34.9 \mathrm{~cm}(13.75 ")$

There were no postnatal complications, and the baby was discharged home with her mother from the newborn nursery after a normal postnatal echocardiogram and renal ultrasound. Chromosome microarray analysis on cord blood failed due to maternal cell contamination. It was ordered again by the infant's pediatrician but was not completed. At 12 months, the patient was non-dysmorphic, growing well, and was on target developmentally. The physical exam was not consistent with 22q11.2 deletion syndrome. A chromosome microarray analysis was offered primarily for reassurance and to rule out any copy number variants, typical or atypical, at the 22q11 locus.

\section{"The arguments for offering NIPT for microdeletion detection to low-risk women are that copy number variants are not associated with advanced maternal age and that microdeletions, as a group, are more prevalent than Down syndrome in infants born to younger mothers."}

\section{Discussion}

These two infants both had a positive cff DNA screening test for 22q11.2 microdeletion during gestation, but only the baby with a prenatally detected cardiac anomaly was affected. This is not surprising as the prior risk for a 22q11.2 microdeletion is substantially higher when a fetus has a cardiac anomaly. In case 2, in spite of her normal development and lack of associated anomalies, the mother of the child with the (presumed) false-positive result was still concerned enough to seek a confirmatory test at a year of age. When an NIPT is positive for 22q11.2 deletion syndrome, a definitive diagnostic test should be offered soon after birth to resolve both the true positives and the false positives.

Chromosome anomalies significantly contribute to the etiology of congenital anomalies in both numerical (aneuploidy) and copy number variants (microdeletions and microduplications). Clinically relevant copy number variants occur in as many as $1.6 \%$ of Readers can also follow EONATOLOGY TO via our Twitter Feed @NEOTODAY 
pregnancies. Increasingly, NIPT, which analyzes maternal serum for fetal (primarily trophoblast) and maternal cell-free DNA, is employed to identify both types of fetal chromosome variants in highrisk and low-risk pregnancies. Although professional societies do not endorse this practice, it is widely offered in clinical practice.

The arguments for offering NIPT for microdeletion detection to low-risk women are that copy number variants are not associated with advanced maternal age and that microdeletions, as a group, are more prevalent than Down syndrome in infants born to younger mothers. Taken together, the 5 most common microdeletions (1p36 deletion, $4 p$ [Wolf-Hirschhorn syndrome], $5 p$ [cri du Chat syndrome], 15q11-13 deletion [Prader-Willi/Angelman syndromes], 22q11.2 deletion [DiGeorge/velocardiofacial syndrome) have an incidence of $1 / 1000$ at birth. This means that a pregnant woman under the age of 29 is more likely to have a child with a microdeletion than a child with Down syndrome.

As with any rare condition, a screening test for microdeletion syndromes is expected to have a high false-positive rate and a low positive predictive value. However, little data has been published on the subject. As more pregnant women choose non-invasive prenatal testing, including microdeletion and standard aneuploidy screening, we can expect more false-positive than true positives. This raises many questions about the most appropriate response to a positive or negative NIPT test for a microdeletion. How worrying is a positive NIPT test? How reassuring is a negative test?

The positive predictive value (PPV) is the ratio of true positives to all positive test results.

Many factors influence the PPV for NIPT for microdeletions, including the prevalence of the disorder in the population, size of the copy number variant, sample characteristics (fetal fraction of DNA, regions of homozygosity within the target), test methodology, and laboratory protocol (SNP coverage, depth of reads). The two main testing methods used for NIPT are not equivalent in their ability to detect $22 q 11.2$ and probably other microdeletions. Lo, et al. (2019) reported a fetus with a confirmed diagnosis of chromosome 22q11.2 deletion in whom two NIPT tests using different methods yielded discordant results. The pregnancy was identified as high-risk by an NIPT test that relied on an SNP-based approach and low-risk by an NIPT test that utilized the genome-wide counting method. This occurrence may be because a high depth of sequencing is required to reliably detect a small microdeletion when a whole-genome approach is used.

A rare disorder's low prevalence means that a positive screening test result is less likely to be a true positive in a low-risk population. This finding is borne out in the general population of pregnant women, in whom the PPV for a positive NIPT microdeletion screen is generally low, ranging from $9-20 \%$. In their study of PPV for NIPT, Chen et al. (2019) found 20 true positives for copy number variants out of 69 with positive NIPT results for a PPV of $28.9 \%$, which, interestingly, was higher than the PPV for trisomy 13 in that study. Petersen et al. (2017) reported Baylor data in which confirmatory testing on 52 positive NIPT screens for microdeletion syndromes revealed $7 / 52$ were true positives, PPV $13.4 \%$. Of these, $6 / 28$ were true positives for $22 q 11.2$ deletion, PPV $21.4 \%$. No indications were given for the original NIPT tests. Among a population of patients tested with NIPT from 7 different laboratories, Schwartz et al. (2018), found 25 confirmed microdeletions in 335 low-risk NIPT positive patients, yielding a PPV of $7.4 \%$ overall with wide confidence limits for each microdeletion type, due to small sample sizes. Of these 25 patients, 1/21 was a true positive for $1 \mathrm{p} 36$ deletion (PPV 4.8\%), 1/6 for $4 p$ deletion (PPV 16.7\%), 6/45 for $5 p$ deletion (PPV 13.3\%), $5 / 80$ for $15 q$ deletion (PPV 6.3\%), and 12/183 for 22q deletion (PPV 6.6\%).

Among the false positives for $15 q$ and $22 q$ microdeletions, Schwartz and colleagues found an over-representation of homozygosity compared to controls, implying that consanguinity between the parents may be a risk factor for false positive NIPT results in these groups, especially when the NIPT test relies on a single nucleic acid polymorphism (SNP) methodology.

As the a priori risk for microdeletion increases in high-risk populations, so does the PPV for a positive NIPT microdeletion test. The presence of fetal anomalies consistent with the diagnosis should increase the PPV substantially. In Chen's report, the PPV was $100 \%$ in the group whose indication for NIPT testing was a fetal structural anomaly on ultrasound. Schwartz et al. reported 7 confirmed microdeletions in their small group of high-risk patients, with indications of a fetal ultrasound abnormality or a family history of microdeletion, yielding a PPV of $43.8 \%$. Helgeson et al. (2015) reported a high PPV for microdeletions detected by NIPT using whole-genome sequencing in a high-risk population. They reported confirmatory studies in 53/55 cases with a positive NIPT for microdeletions. Among NIPT tests positive for a $22 q 11.2$ deletion, 23/32 were confirmed in the mother, the fetus, or both for a PPV of $71.9 \%$. However, the authors expected 44 affected cases with $22 q 11.2$ deletion in this cohort, and they estimated the sensitivity of the test to be $70.5 \%$. Among those NIPT tests that were positive for $15 q, 8 / 9$ were confirmed for a PPV of $88.9 \%$. These authors report that in those samples found to have a microdeletion, a fetal ultrasound finding was the most common indication for the NIPT test $(48.2 \%)$.

To address how reassuring a negative NIPT with microdeletion detection for 22q11.2 would be, Asoglu et al. (2020) examined a cohort of patients with congenital heart defects whose cytogenetic diagnosis had been established. In their retrospective analysis of 302 CHD cases with diagnostic genetic results, 98/302 had a confirmed cytogenetic abnormality. Of these, $31 / 98(31.6 \%)$ or $10.3 \%$ of the total group would not have been detectable by NIPT for aneuploidy or $22 q 11.2$ microdeletion analysis. This reinforces the need for cytogenetic studies in newborns with CHD who have had a negative NIPT that included microdeletion analysis.

The two cases above, one with a prenatally apparent cardiac

The only worldwide monthly publication
exclusively serving Pediatric and Adult
Cardiologists that focus on Congenital/
$\begin{aligned} & \text { CONGENITAL } \\ & \text { CARDIOLOGY } \\ & \text { TODAY }\end{aligned}$
Cardiothoractural Heart Disease (CHD), and


anomaly and one without, illustrate how the likelihood of a true positive result varies with the indication for NIPT testing. Congenital heart defects (CHD) are the most common birth defect, affecting almost $1 \%$ of all live-born infants. The $22 q 11.2$ deletion syndrome is the most common microdeletion in the newborn, with a prevalence of 1 in 4000 live births. The presence of a CHD will substantially increase the chance that a positive NIPT for $22 q 11.2$ is a true positive. Without a fetal anomaly, the same positive test is more likely to be a false positive. As NIPT testing for microdeletions is offered to low-risk and high-risk women alike, medical providers caring for these infants should consider the indication for the NIPT test in the first place to understand its likely significance to their patient. In any event, a confirmatory test is warranted, if only for reassurance.

Practical Applications:

1. Cell-free fetal DNA is a screening test that should not be considered definitive or diagnostic.

2. A positive NIPT for a microdeletion has a higher PPV when the indication for testing was a fetal structural anomaly detected by ultrasound

3. Confirm any positive NIPT test with chromosome analysis (for aneuploidy) or chromosome microarray (for copy number variants).

4. Do not let a negative NIPT test dissuade you from ordering a definitive chromosome study when the phenotype suggests a microdeletion syndrome.

References:

Asoglu MR, Cutting EM, Ozdemir $\mathrm{H}$, et al. The rate of undetectable genetic causes by Cell-free DNA test in congenital heart defects. J Matern Fetal Neonatal Med. 2020 Apr;26:1-7. PMID: 32338089

Chen $Y, Y u Q$, Mao X, et al. Non-invasive prenatal testing for chromosome aneuploidies and subchromosomal microdeletions/ microduplications in a cohort of 42,910 single pregnancies with different clinical features. Hum Genomics. 2019 Nov 29;13(1):60. PMID: 31783780

Guy C, Haji-Sheikhi F, Rowland CM, et al. Prenatal cell-free DNA screening for fetal aneuploidy in pregnant women at average or high risk: Results from a large US clinical laboratory. Mol Genet Genomic Med. 2019;7(3):e545. PMID: 30706702

Helgeson J, Wardrop J, Boomer T, et al. Clinical outcome of subchromosomal events detected by whole-genome non-invasive prenatal testing. Prenat Diagn. 2015 Oct;35(10):9991004. PMID: 26088833

Lo LM, Shiau CS, Chen KC, et al. Screening for 22q11.2 deletion syndrome by two non-invasive prenatal testing methodologies: A case with discordant results. Taiwan J Obstet Gynecol. 2019 Jan;58(1):40-42. PMID: 30638477

Petersen AK, Cheung SW, Smith JL, et al. Positive predictive value estimates for cell-free non-invasive prenatal screening from data of a large referral genetic diagnostic laboratory. Am J Obstet Gynecol. 2017 Dec;217(6):691.e1-691.e6. PMID: 29032050

Ravi H, McNeill G, Goel S, et al. Validation of a SNP-based noninvasive prenatal test to detect the fetal 22q11.2 deletion in maternal plasma samples. PLoS One. 2018;13(2):e0193476. PMID: 29474437

Schwartz S, Kohan M, Pasion R, et al. Clinical experience of laboratory follow-up with non-invasive prenatal testing using cell- free DNA and positive microdeletion results in 349 cases. Prenat Diagn. 2018 Feb;38(3):210-218. PMID: 29338128

Disclosures: The authors have no relevant disclosures.

NT
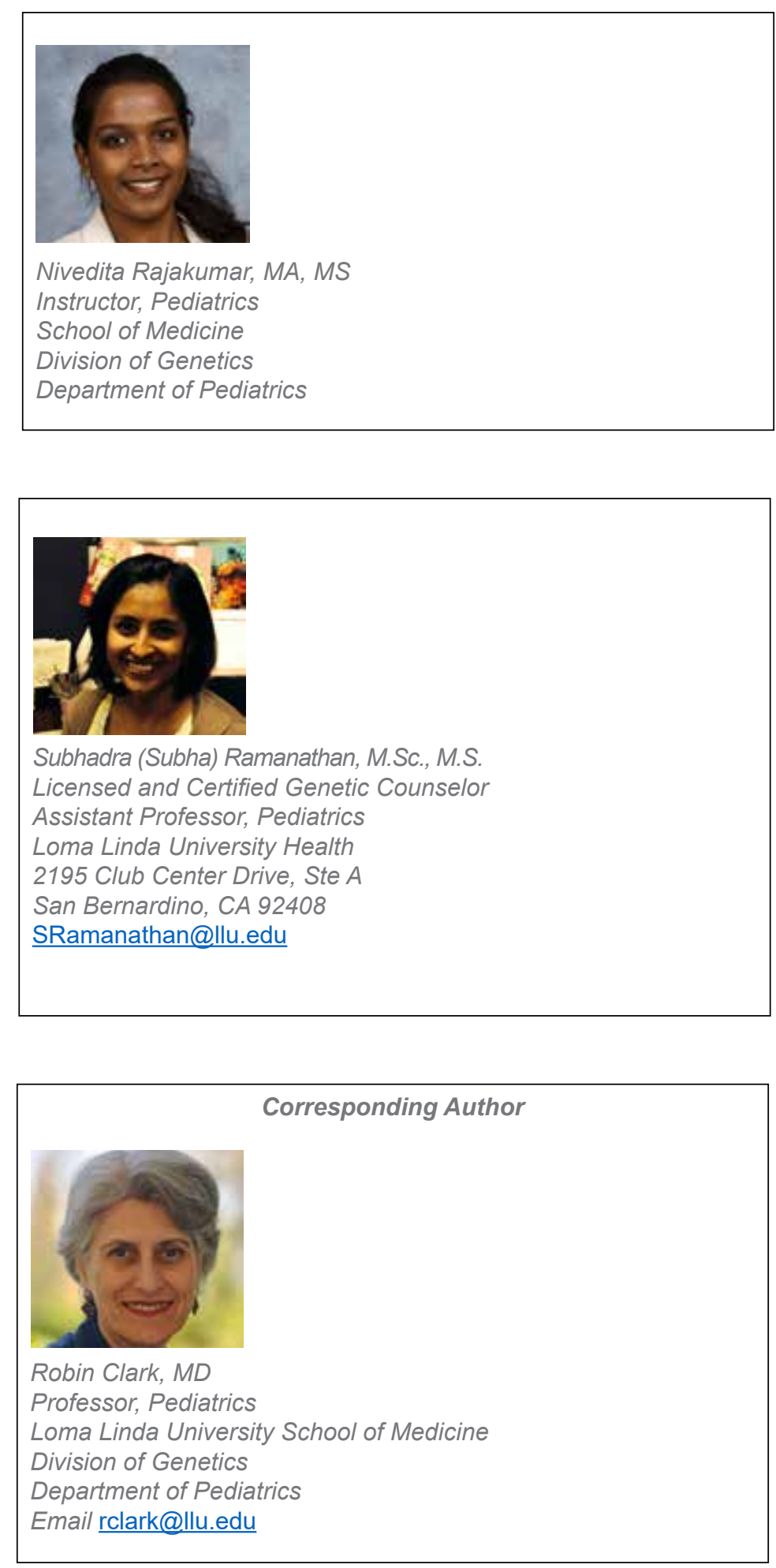